\title{
Weighted composition followed and proceeded by differentiation operators from Zygmund spaces to Bloch-type spaces
}

Jianren Long ${ }^{1,2^{*}}$, Chunhui Qiu' and Pengcheng Wu${ }^{2}$

"Correspondence:

longjianren2004@163.com

'School of Mathematical Sciences,

Xiamen University, Xiamen, 361005,

P.R. China

${ }^{2}$ School of Mathematics and

Computer Science, Guizhou Normal

University, Guiyang, 550001,

P.R. China

\begin{abstract}
The boundedness and compactness of the weighted composition followed and proceeded by differentiation operators from Zygmund spaces to Bloch-type spaces and little Bloch-type spaces are characterized.

MSC: 47B38; 30H30; 32C15
\end{abstract}

Keywords: Zygmund spaces; Bloch-type spaces; weighted composition followed and proceeded by differentiation operators; boundedness; compactness

\section{Introduction}

Let $\Delta=\{z:|z|<1\}$ be the open unit disc in the complex plane $\mathbb{C}$, and let $H(\Delta)$ be the class of all analytic functions on $\Delta$. The $\alpha$-Bloch space $B^{\alpha}(0<\alpha<\infty)$ is, by definition, the set of all functions $f$ in $H(\Delta)$ such that

$$
\|f\|_{B^{\alpha}}=|f(0)|+\sup _{z \in \Delta}\left(1-|z|^{2}\right)^{\alpha}\left|f^{\prime}(z)\right|<\infty .
$$

Under the above norm, $B^{\alpha}$ is a Banach space. When $\alpha=1, B^{1}=B$ is the well-known Bloch space. Let $B_{0}^{\alpha}$ denote the subspace of $B^{\alpha}$, i.e.,

$$
B_{0}^{\alpha}=\left\{f:\left(1-|z|^{2}\right)^{\alpha}\left|f^{\prime}(z)\right| \rightarrow 0 \text { as }|z| \rightarrow 1, f \in B^{\alpha}\right\} .
$$

This space is called the little $\alpha$-Bloch space (see [1]).

Assume that $\mu$ is a positive continuous function on $[0,1)$, having the property that there exist positive numbers $s$ and $t, 0<s<t$, and $\delta \in[0,1)$, such that

$$
\begin{aligned}
& \frac{\mu(r)}{(1-r)^{s}} \text { is decreasing on }[\delta, 1), \lim _{r \rightarrow 1} \frac{\mu(r)}{(1-r)^{s}}=0, \\
& \frac{\mu(r)}{(1-r)^{t}} \text { is increasing on }[\delta, 1), \lim _{r \rightarrow 1} \frac{\mu(r)}{(1-r)^{t}}=\infty \text {. }
\end{aligned}
$$

Then $\mu$ is called a normal function (see [2]).

Denote (see, e.g., [3-5])

$$
B_{\mu}=\left\{f:\|f\|_{B_{\mu}}=|f(0)|+\sup _{z \in \Delta} \mu(|z|)\left|f^{\prime}(z)\right|<\infty, f \in H(\Delta)\right\} .
$$

(2014 Long et al:; licensee Springer. This is an Open Access article distributed under the terms of the Creative Commons Attribution License (http://creativecommons.org/licenses/by/2.0), which permits unrestricted use, distribution, and reproduction in any medium, provided the original work is properly cited. 
It is well known that $B_{\mu}$ is a Banach space with the norm $\|\cdot\|_{B_{\mu}}$ (see [4]).

Let $B_{\mu, 0}$ denote the subspace of $B_{\mu}$, i.e.,

$$
B_{\mu, 0}=\left\{f: \mu(|z|)\left|f^{\prime}(z)\right| \rightarrow 0 \text { as }|z| \rightarrow 1, f \in B_{\mu}\right\} .
$$

This space is called the little Bloch-type space. When $\mu(r)=\left(1-r^{2}\right)^{\alpha}$, the induced space $B_{\mu}$ becomes the $\alpha$-Bloch space $B^{\alpha}$.

An $f$ in $H(\Delta)$ is said to belong to the Zygmund space, denoted by $\mathcal{Z}$, if

$$
\sup \frac{\left|f\left(e^{i(\theta+h)}\right)+f\left(e^{i(\theta-h)}\right)-2 f\left(e^{i \theta}\right)\right|}{h}<\infty
$$

where the supremum is taken over all $e^{i \theta} \in \partial \Delta$ and $h>0$. By Theorem 5.3 in [6], we see that $f \in \mathcal{Z}$ if and only if

$$
\|f\|_{\mathbb{Z}}=|f(0)|+\left|f^{\prime}(0)\right|+\sup _{z \in \Delta}\left(1-|z|^{2}\right)\left|f^{\prime \prime}(z)\right|<\infty .
$$

It is easy to check that $\mathcal{Z}$ is a Banach space under the above norm. For every $f \in \mathcal{Z}$, by using a result in [7], we have

$$
\left|f^{\prime}(z)\right| \leq C\|f\|_{\mathcal{Z}} \ln \frac{e}{1-|z|^{2}} .
$$

Let $\mathcal{Z}_{0}$ denote the subspace of $\mathcal{Z}$ consisting of those $f \in \mathcal{Z}$ for which

$$
\lim _{|z| \rightarrow 1}\left(1-|z|^{2}\right)\left|f^{\prime \prime}(z)\right|=0
$$

The space $\mathcal{Z}_{0}$ is called the little Zygmund space. For the corresponding $n$-dimensional Zygmund space see, e.g., [8] and [9].

Let $\varphi$ be a nonconstant analytic self-map of $\Delta$, and let $\phi$ be an analytic function in $\Delta$. We define the linear operators

$$
\phi C_{\varphi} D f=\phi\left(f^{\prime} \circ \varphi\right)=\phi f^{\prime}(\varphi) \quad \text { and } \quad \phi D C_{\varphi} f=\phi(f \circ \varphi)^{\prime}=\phi f^{\prime}(\varphi) \varphi^{\prime}, \quad \text { for } f \in H(\Delta) .
$$

They are called weighted composition followed and proceeded by differentiation operators, respectively, where $C_{\varphi}$ and $D$ are composition and differentiation operators respectively. Associated with $\varphi$ is the composition operator $C_{\varphi} f=f \circ \varphi$ and weighted composition operator $\phi C_{\varphi} f=\phi f \circ \varphi$ for $\phi \in H(\Delta)$ and $f \in H(\Delta)$. It is interesting to provide a function theoretic characterization for $\varphi$ inducing a bounded or compact composition operator, weighted composition operator and related ones on various spaces (see, e.g., [1019]). For example, it is well known that $C_{\varphi}$ is bounded on the classical Hardy, Bloch and Bergman spaces. Operators $D C_{\varphi}$ and $C_{\varphi} D$ as well as some other products of linear operators were studied, for example, in [20-29] (see also the references therein). There has been some considerable recent interest in investigation various type of operators from or to Zygmund type spaces (see, [7, 11, 23, 30-37]).

In this paper, we investigate the operators $\phi D C_{\varphi}$ and $\phi C_{\varphi} D$ from Zygmund spaces to Bloch-type spaces and little Bloch-type spaces. Some sufficient and necessary conditions for the boundedness and compactness of these operators are given. 
Throughout this paper, constants are denoted by $C$, they are positive and may differ from one occurrence to the other. The notation $A \approx B$ means that there is a positive constant $C$ such that $\frac{B}{C} \leq A \leq C B$.

\section{Main results and proofs}

In this section, we state and prove our main results. In order to formulate our main results, we quote several lemmas which will be used in the proofs of the main results in this paper. The following lemma can be proved in a standard way (see, e.g., Proposition 3.11 in [10]). Hence we omit the details.

Lemma 2.1 Let $\varphi$ be an analytic self-map of $\Delta$, and let $\phi$ be an analytic function in $\Delta$. Suppose that $\mu$ is normal. Then $\phi D C_{\varphi}\left(\right.$ or $\left.\phi C_{\varphi} D\right): \mathcal{Z}\left(\right.$ or $\left.\mathcal{Z}_{0}\right) \rightarrow B_{\mu}$ is compact if and only if $\phi D C_{\varphi}\left(\right.$ or $\left.\phi C_{\varphi} D\right): \mathcal{Z}\left(\right.$ or $\left.\mathcal{Z}_{0}\right) \rightarrow B_{\mu}$ is bounded and for any bounded sequence $\left\{f_{n}\right\}_{n \in N}$ in $\mathcal{Z}$ (or $\mathcal{Z}_{0}$ ) which converges to zero uniformly on compact subsets of $\Delta$ as $n \rightarrow \infty$, and $\left\|\phi D C_{\varphi} f_{n}\right\|_{B_{\mu}} \rightarrow 0\left(\right.$ or $\left.\left\|\phi C_{\varphi} D f_{n}\right\|_{B_{\mu}} \rightarrow 0\right)$ as $n \rightarrow \infty$.

Lemma 2.2 [25] A closed set $\mathbb{K}$ of $B_{\mu, 0}$ is compact if and only if it is bounded and satisfied

$$
\lim _{|z| \rightarrow 1} \sup _{f \in \mathbb{K}} \mu(|z|)\left|f^{\prime}(z)\right|=0 .
$$

Theorem 2.3 Let $\varphi$ be an analytic self-map of $\Delta$, and let $\phi$ be an analytic function in $\Delta$. Suppose that $\mu$ is normal. Then the following statements are equivalent.

(i) $\phi D C_{\varphi}: \mathcal{Z} \rightarrow B_{\mu}$ is bounded;

(ii) $\phi D C_{\varphi}: \mathcal{Z}_{0} \rightarrow B_{\mu}$ is bounded;

(iii)

$$
\sup _{z \in \Delta} \mu(|z|) \frac{\left|\phi(z)\left(\varphi^{\prime}(z)\right)^{2}\right|}{1-|\varphi(z)|^{2}}<\infty
$$

and

$$
\sup _{z \in \Delta} \mu(|z|)\left|\phi(z) \varphi^{\prime \prime}(z)+\phi^{\prime}(z) \varphi^{\prime}(z)\right| \ln \frac{e}{1-|\varphi(z)|^{2}}<\infty .
$$

Proof of Theorem 2.3 (i) $\Rightarrow$ (ii). This implication is obvious.

(ii) $\Rightarrow$ (iii). Assume that $\phi D C_{\varphi}: \mathcal{Z}_{0} \rightarrow B_{\mu}$ is bounded, i.e., there exists a constant $C$ such that

$$
\left\|\phi D C_{\varphi} f\right\|_{B_{\mu}} \leq C\|f\|_{\mathcal{Z}}
$$

for all $f \in \mathcal{Z}_{0}$. Taking the functions $f(z)=z \in \mathcal{Z}_{0}$ and $f(z)=z^{2} \in \mathcal{Z}_{0}$ respectively, we get

$$
\begin{aligned}
& \sup _{z \in \Delta} \mu(|z|)\left|\phi(z) \varphi^{\prime \prime}(z)+\phi^{\prime}(z) \varphi^{\prime}(z)\right|<\infty, \\
& \sup _{z \in \Delta} \mu(|z|)\left|\left(\phi(z) \varphi^{\prime \prime}(z)+\phi^{\prime}(z) \varphi^{\prime}(z)\right) \varphi(z)+\phi(z)\left(\varphi^{\prime}(z)\right)^{2}\right|<\infty .
\end{aligned}
$$

Using these facts and the boundedness of function $\varphi$, we have

$$
\sup _{z \in \Delta} \mu(|z|)\left|\phi(z)\left(\varphi^{\prime}(z)\right)^{2}\right|<\infty .
$$


Set

$$
h(z)=(z-1)\left[\left(1+\ln \frac{1}{1-z}\right)^{2}+1\right]
$$

and

$$
h_{a}(z)=\frac{h(\bar{a} z)}{\bar{a}}\left(\ln \frac{1}{1-|a|^{2}}\right)^{-1}
$$

for $a \in \Delta \backslash\{0\}$. It is known that $h_{a} \in \mathcal{Z}_{0}$ (see [7]). Since

$$
h_{a}^{\prime}(z)=\left(\ln \frac{1}{1-\bar{a} z}\right)^{2}\left(\ln \frac{1}{1-|a|^{2}}\right)^{-1}
$$

and

$$
h_{a}^{\prime \prime}(z)=\frac{2 \bar{a}}{1-\bar{a} z}\left(\ln \frac{1}{1-\bar{a} z}\right)\left(\ln \frac{1}{1-|a|^{2}}\right)^{-1},
$$

for $|\varphi(\lambda)|>\frac{1}{2}$, we have

$$
\begin{aligned}
C\left\|\phi D C_{\varphi}\right\|_{\mathcal{Z}_{0} \rightarrow B_{\mu}} \geq & \left\|\phi D C_{\varphi} h_{\varphi(\lambda)}\right\|_{B_{\mu}} \geq \mu(|\lambda|)\left|\phi(\lambda) \varphi^{\prime \prime}(\lambda)+\phi^{\prime}(\lambda) \varphi^{\prime}(\lambda)\right| \ln \frac{1}{1-|\varphi(\lambda)|^{2}} \\
& -2 \mu(|\lambda|) \frac{\left|\phi(\lambda)\left(\varphi^{\prime}(\lambda)\right)^{2} \varphi(\lambda)\right|}{1-|\varphi(\lambda)|^{2}} .
\end{aligned}
$$

Hence

$$
\begin{aligned}
& \mu(|\lambda|)\left|\phi(\lambda) \varphi^{\prime \prime}(\lambda)+\phi^{\prime}(\lambda) \varphi^{\prime}(\lambda)\right| \ln \frac{1}{1-|\varphi(\lambda)|^{2}} \\
& \quad \leq C\left\|\phi D C_{\varphi}\right\|_{\mathcal{Z}_{0} \rightarrow B_{\mu}}+2 \mu(|\lambda|) \frac{\left|\phi(\lambda)\left(\varphi^{\prime}(\lambda)\right)^{2} \varphi(\lambda)\right|}{1-|\varphi(\lambda)|^{2}} .
\end{aligned}
$$

For $a \in \Delta \backslash\{0\}$, set

$$
f_{a}(z)=\frac{h(\bar{a} z)}{\bar{a}}\left(\ln \frac{1}{1-|a|^{2}}\right)^{-1}-\int_{0}^{z} \ln \frac{1}{1-\bar{a} \omega} d \omega .
$$

Then $f_{a} \in \mathcal{Z}_{0}$. It is easy to see that

$$
f_{a}^{\prime}(z)=\left(\ln \frac{1}{1-\bar{a} z}\right)^{2}\left(\ln \frac{1}{1-|a|^{2}}\right)^{-1}-\ln \frac{1}{1-\bar{a} z}, \quad f_{a}^{\prime}(a)=0,
$$

and

$$
f_{a}^{\prime \prime}(z)=\frac{2 \bar{a}}{1-\bar{a} z}\left(\ln \frac{1}{1-\bar{a} z}\right)\left(\ln \frac{1}{1-|a|^{2}}\right)^{-1}-\frac{\bar{a}}{1-\bar{a} z}, \quad f_{a}^{\prime \prime}(a)=\frac{\bar{a}}{1-|a|^{2}} .
$$

Therefore

$$
C\left\|\phi D C_{\varphi}\right\|_{\mathcal{Z}_{0} \rightarrow B_{\mu}} \geq\left\|\phi D C_{\varphi} f_{\varphi(\lambda)}\right\|_{B_{\mu}} \geq \mu(|\lambda|) \frac{\left|\phi(\lambda)\left(\varphi^{\prime}(\lambda)\right)^{2} \varphi(\lambda)\right|}{1-|\varphi(\lambda)|^{2}} .
$$


From (2.8) and (2.10), we have

$$
\sup _{|\varphi(\lambda)|>\frac{1}{2}} \mu(|\lambda|)\left|\phi(\lambda) \varphi^{\prime \prime}(\lambda)+\phi^{\prime}(\lambda) \varphi^{\prime}(\lambda)\right| \ln \frac{1}{1-|\varphi(\lambda)|^{2}} \leq C\left\|\phi D C_{\varphi}\right\|_{\mathcal{Z}_{0} \rightarrow B_{\mu}}<\infty
$$

On the other hand, from the first inequality in (2.3), we have

$$
\begin{aligned}
& \sup _{|\varphi(\lambda)| \leq \frac{1}{2}} \mu(|\lambda|)\left|\phi(\lambda) \varphi^{\prime \prime}(\lambda)+\phi^{\prime}(\lambda) \varphi^{\prime}(\lambda)\right| \ln \frac{1}{1-|\varphi(\lambda)|^{2}} \\
& \quad \leq \sup _{\lambda \in \Delta} \mu(|\lambda|)\left|\phi(\lambda) \varphi^{\prime \prime}(\lambda)+\phi^{\prime}(\lambda) \varphi^{\prime}(\lambda)\right| \ln \frac{4}{3}<\infty .
\end{aligned}
$$

Hence, from (2.3), (2.11), and (2.12), we obtain (2.2). Further, from (2.10), we have

$$
\begin{aligned}
\sup _{|\varphi(\lambda)|>\frac{1}{2}} \mu(|\lambda|) \frac{\left|\phi(\lambda)\left(\varphi^{\prime}(\lambda)\right)^{2}\right|}{1-|\varphi(\lambda)|^{2}} & \leq \sup _{|\varphi(\lambda)|>\frac{1}{2}} 2 \mu(|\lambda|) \frac{\left|\phi(\lambda)\left(\varphi^{\prime}(\lambda)\right)^{2} \varphi(\lambda)\right|}{1-|\varphi(\lambda)|^{2}} \\
& \leq C\left\|\phi D C_{\varphi}\right\|_{\mathcal{Z}_{0} \rightarrow B_{\mu}}<\infty
\end{aligned}
$$

On the other hand, by (2.4), we have

$$
\sup _{|\varphi(\lambda)| \leq \frac{1}{2}} \mu(|\lambda|) \frac{\left|\phi(\lambda)\left(\varphi^{\prime}(\lambda)\right)^{2}\right|}{1-|\varphi(\lambda)|^{2}} \leq \sup _{|\varphi(\lambda)| \leq \frac{1}{2}} \frac{4}{3} \mu(|\lambda|)\left|\phi(\lambda)\left(\varphi^{\prime}(\lambda)\right)^{2}\right|<\infty .
$$

Combining (2.13) and (2.14), (2.1) follows.

(iii) $\Rightarrow$ (i). Assume that (2.1) and (2.2) hold. Then, for every $f \in \mathcal{Z}$, from (1.4), we have

$$
\begin{aligned}
\mu(|z|)\left|\left(\phi D C_{\varphi} f\right)^{\prime}(z)\right| \\
=\mu(|z|)\left|\phi^{\prime}(z) \varphi^{\prime}(z) f^{\prime}(\varphi(z))+\phi(z)\left[f^{\prime \prime}(\varphi(z))\left(\varphi^{\prime}(z)\right)^{2}+f^{\prime}(\varphi(z)) \varphi^{\prime \prime}(z)\right]\right| \\
\leq \mu(|z|)\left|\phi(z) f^{\prime \prime}(\varphi(z))\left(\varphi^{\prime}(z)\right)^{2}\right|+\mu(|z|) \mid\left[\phi(z) \varphi^{\prime \prime}(z)+\phi^{\prime}(z) \varphi^{\prime}(z)\right] f^{\prime}(\varphi(z) \mid \\
\leq \mu(|z|) C \frac{\left|\phi(z)\left(\varphi^{\prime}(z)\right)^{2}\right|}{1-|\varphi(z)|^{2}}\|f\|_{\mathcal{Z}}+\mu(|z|) C\left|\phi(z) \varphi^{\prime \prime}(z)+\phi^{\prime}(z) \varphi^{\prime}(z)\right| \\
\quad \times \ln \frac{e}{1-|\varphi(z)|^{2}}\|f\|_{\mathcal{Z}} .
\end{aligned}
$$

Taking the supremum in (2.15) for $z \in \Delta$, and employing (2.1) and (2.2), we deduce that $\phi D C_{\varphi}: \mathcal{Z} \rightarrow B_{\mu}$ is bounded. The proof of Theorem 2.3 is completed.

Theorem 2.4 Let $\varphi$ be an analytic self-map of $\Delta$, and let $\phi$ be an analytic function in $\Delta$. Suppose that $\mu$ is normal. Then the following statements are equivalent.

(i) $\phi D C_{\varphi}: \mathcal{Z} \rightarrow B_{\mu}$ is compact;

(ii) $\phi D C_{\varphi}: \mathcal{Z}_{0} \rightarrow B_{\mu}$ is compact;

(iii) $\phi D C_{\varphi}: \mathcal{Z} \rightarrow B_{\mu}$ is bounded,

$$
\lim _{|\varphi(z)| \rightarrow 1} \mu(|z|) \frac{\left|\phi(z)\left(\varphi^{\prime}(z)\right)^{2}\right|}{1-|\varphi(z)|^{2}}=0
$$


and

$$
\lim _{|\varphi(z)| \rightarrow 1} \mu(|z|)\left|\phi(z) \varphi^{\prime \prime}(z)+\phi^{\prime}(z) \varphi^{\prime}(z)\right| \ln \frac{e}{1-|\varphi(z)|^{2}}=0 .
$$

Proof of Theorem 2.4 (i) $\Rightarrow$ (ii). This implication is clear.

(ii) $\Rightarrow$ (iii). Assume that $\phi D C_{\varphi}: \mathcal{Z}_{0} \rightarrow B_{\mu}$ is compact. Then it is clear that $\phi D C_{\varphi}: \mathcal{Z}_{0} \rightarrow$ $B_{\mu}$ is bounded. By Theorem 2.3 we know that $\phi D C_{\varphi}: \mathcal{Z} \rightarrow B_{\mu}$ is bounded. Let $\left(z_{n}\right)_{n \in N}$ be a sequence in $\Delta$ such that $\left|\varphi\left(z_{n}\right)\right| \rightarrow 1$ as $n \rightarrow \infty$ and $\varphi\left(z_{n}\right) \neq 0, n \in N$ (if such a sequence does not exist then (2.16) and (2.17) are vacuously satisfied). Set

$$
h_{n}(z)=\frac{h\left(\overline{\varphi\left(z_{n}\right)} z\right)}{\overline{\varphi\left(z_{n}\right)}}\left(\ln \frac{1}{1-\left|\varphi\left(z_{n}\right)\right|^{2}}\right)^{-1}, \quad n \in N .
$$

Then from the proof of Theorem 2.3, we see that $h_{n} \in \mathcal{Z}_{0}$ for each $n \in N$. Moreover $h_{n} \rightarrow 0$ uniformly on compact subsets of $\Delta$ as $n \rightarrow \infty$ and

$$
h_{n}^{\prime}\left(\varphi\left(z_{n}\right)\right)=\ln \frac{1}{1-\left|\varphi\left(z_{n}\right)\right|^{2}}, \quad h_{n}^{\prime \prime}\left(\varphi\left(z_{n}\right)\right)=\ln \frac{2 \overline{\varphi\left(z_{n}\right)}}{1-\left|\varphi\left(z_{n}\right)\right|^{2}} .
$$

Since $\phi D C_{\varphi}: \mathcal{Z}_{0} \rightarrow B_{\mu}$ is compact, by Lemma 2.1, we have

$$
\lim _{n \rightarrow \infty}\left\|\phi D C_{\varphi} h_{n}\right\|_{B_{\mu}}=0 .
$$

On the other hand, similar to the proof of Theorem 2.3, we have

$$
\begin{aligned}
\left\|\phi D C_{\varphi} h_{n}\right\|_{B_{\mu}} \geq & \left|2 \mu\left(\left|z_{n}\right|\right) \frac{\left|\phi\left(z_{n}\right)\left(\varphi^{\prime}\left(z_{n}\right)\right)^{2}\right|\left|\varphi\left(z_{n}\right)\right|}{1-\left|\varphi\left(z_{n}\right)\right|^{2}}-\mu\left(\left|z_{n}\right|\right)\right| \\
& \times \phi\left(z_{n}\right) \varphi^{\prime \prime}\left(z_{n}\right)+\phi^{\prime}\left(z_{n}\right) \varphi^{\prime}\left(z_{n}\right)\left|\ln \frac{1}{1-\left|\varphi\left(z_{n}\right)\right|^{2}}\right|,
\end{aligned}
$$

which implies that

$$
\begin{aligned}
& \lim _{n \rightarrow \infty} 2 \mu\left(\left|z_{n}\right|\right) \frac{\left|\phi\left(z_{n}\right)\left(\varphi^{\prime}\left(z_{n}\right)\right)^{2}\right|\left|\varphi\left(z_{n}\right)\right|}{1-\left|\varphi\left(z_{n}\right)\right|^{2}} \\
& \quad=\lim _{n \rightarrow \infty} \mu\left(\left|z_{n}\right|\right)\left|\phi\left(z_{n}\right) \varphi^{\prime \prime}\left(z_{n}\right)+\phi^{\prime}\left(z_{n}\right) \varphi^{\prime}\left(z_{n}\right)\right| \ln \frac{1}{1-\left|\varphi\left(z_{n}\right)\right|^{2}},
\end{aligned}
$$

if one of these two limits exists.

Next, set

$$
\begin{aligned}
f_{n}(z)= & \frac{h\left(\overline{\varphi\left(z_{n}\right)} z\right)}{\overline{\varphi\left(z_{n}\right)}}\left(\ln \frac{1}{1-\left|\varphi\left(z_{n}\right)\right|^{2}}\right)^{-1} \\
& -\int_{0}^{z} \ln ^{3} \frac{1}{1-\overline{\varphi\left(z_{n}\right)} \omega} d \omega\left(\ln \frac{1}{1-\left|\varphi\left(z_{n}\right)\right|^{2}}\right)^{-2} .
\end{aligned}
$$

Then $f_{n} \in \mathcal{Z}_{0}$ and $f_{n}$ converges to 0 uniformly on compact subsets of $\Delta$ as $n \rightarrow \infty$ (see [7]). Since

$$
f_{n}^{\prime}(z)=\left(\ln \frac{1}{1-\overline{\varphi\left(z_{n}\right)} z}\right)^{2}\left(\ln \frac{1}{1-\left|\varphi\left(z_{n}\right)\right|^{2}}\right)^{-1}-\left(\ln \frac{1}{1-\overline{\varphi\left(z_{n}\right)} z}\right)^{3}\left(\ln \frac{1}{1-\left|\varphi\left(z_{n}\right)\right|^{2}}\right)^{-2}
$$


we have $f_{n}^{\prime}\left(\varphi\left(z_{n}\right)\right)=0$, for every $n \in N$ and

$$
f_{n}^{\prime \prime}\left(\varphi\left(z_{n}\right)\right)=-\frac{\overline{\varphi\left(z_{n}\right)}}{1-\left|\varphi\left(z_{n}\right)\right|^{2}} .
$$

By using these facts, since $\phi D C_{\varphi}: \mathcal{Z}_{0} \rightarrow B_{\mu}$ is compact, and from Lemma 2.1, we find that

$$
0 \leq \lim _{n \rightarrow \infty} \mu\left(\left|z_{n}\right|\right) \frac{\left|\phi\left(z_{n}\right)\left(\varphi^{\prime}\left(z_{n}\right)\right)^{2}\right|\left|\varphi\left(z_{n}\right)\right|}{1-\left|\varphi\left(z_{n}\right)\right|^{2}} \leq \lim _{n \rightarrow \infty}\left\|\phi D C_{\varphi} f_{n}\right\|_{B_{\mu}}=0 .
$$

Therefore

$$
\lim _{n \rightarrow \infty} \mu\left(\left|z_{n}\right|\right) \frac{\left|\phi\left(z_{n}\right)\left(\varphi^{\prime}\left(z_{n}\right)\right)^{2}\right|}{1-\left|\varphi\left(z_{n}\right)\right|^{2}}=\lim _{n \rightarrow \infty} \mu\left(\left|z_{n}\right|\right) \frac{\left|\phi\left(z_{n}\right)\left(\varphi^{\prime}\left(z_{n}\right)\right)^{2} \varphi\left(z_{n}\right)\right|}{1-\left|\varphi\left(z_{n}\right)\right|^{2}}=0,
$$

which implies (2.16). From this and (2.19), we have

$$
\lim _{n \rightarrow \infty} \mu\left(\left|z_{n}\right|\right)\left|\phi\left(z_{n}\right) \varphi^{\prime \prime}\left(z_{n}\right)+\phi^{\prime}\left(z_{n}\right) \varphi^{\prime}\left(z_{n}\right)\right| \ln \frac{1}{1-\left|\varphi\left(z_{n}\right)\right|^{2}}=0 .
$$

From (2.21), it follows that $\lim _{n \rightarrow \infty} \mu\left(\left|z_{n}\right|\right)\left|\phi\left(z_{n}\right) \varphi^{\prime \prime}\left(z_{n}\right)+\phi^{\prime}\left(z_{n}\right) \varphi^{\prime}\left(z_{n}\right)\right|=0$, which altogether imply (2.17).

(iii) $\Rightarrow$ (i). Suppose that $\phi D C_{\varphi}: \mathcal{Z} \rightarrow B_{\mu}$ is bounded and conditions (2.16) and (2.17) hold. From Theorem 2.3, it follows that

$$
\begin{aligned}
& C_{1}=\sup _{z \in \Delta} \mu(|z|)\left|\phi(z) \varphi^{\prime \prime}(z)+\phi^{\prime}(z) \varphi^{\prime}(z)\right|<\infty, \\
& C_{2}=\sup _{z \in \Delta} \mu(|z|)\left|\phi(z)\left(\varphi^{\prime}(z)\right)^{2}\right|<\infty .
\end{aligned}
$$

By the assumption, for every $\varepsilon>0$, there is a $\delta \in(0,1)$, such that

$$
\begin{aligned}
& \mu(|z|) \frac{\left|\phi(z)\left(\varphi^{\prime}(z)\right)^{2}\right|}{1-|\varphi(z)|^{2}}<\varepsilon \text { and } \\
& \mu(|z|)\left|\phi(z) \varphi^{\prime \prime}(z)+\phi^{\prime}(z) \varphi^{\prime}(z)\right| \ln \frac{e}{1-|\varphi(z)|^{2}}<\varepsilon,
\end{aligned}
$$

whenever $\delta<|\varphi(z)|<1$.

Assume that $\left(f_{k}\right)_{k \in N}$ is a sequence in $\mathcal{Z}$ such that $\sup _{k \in N}\left\|f_{k}\right\|_{\mathbb{Z}} \leq L$ and $f_{k}$ converges to 0 uniformly on compact subsets of $\Delta$ as $k \rightarrow \infty$. Let $K=\{z \in \Delta:|\varphi(z)| \leq \delta\}$. Then by (1.4), (2.22), and (2.23), we have

$$
\begin{aligned}
\sup _{z \in \Delta} \mu(|z|)\left|\left(\phi D C_{\varphi} f_{k}\right)^{\prime}(z)\right| \\
=\sup _{z \in \Delta} \mu(|z|)\left|\phi^{\prime}(z) \varphi^{\prime}(z) f_{k}^{\prime}(\varphi(z))+\phi(z)\left[f_{k}^{\prime \prime}(\varphi(z))\left(\varphi^{\prime}(z)\right)^{2}+f_{k}^{\prime}(\varphi(z)) \varphi^{\prime \prime}(z)\right]\right| \\
\leq \sup _{z \in \Delta} \mu(|z|)\left|\phi(z)\left(\varphi^{\prime}(z)\right)^{2} f^{\prime \prime}(\varphi(z))\right|+\sup _{z \in \Delta} \mu(|z|)\left|\left[\phi(z) \varphi^{\prime \prime}(z)+\phi^{\prime}(z) \varphi^{\prime}(z)\right] f^{\prime}(\varphi(z))\right| \\
\leq \sup _{z \in K} \mu(|z|)\left|\phi(z)\left(\varphi^{\prime}(z)\right)^{2} f^{\prime \prime}(\varphi(z))\right|+\sup _{z \in K} \mu(|z|)\left|\left[\phi(z) \varphi^{\prime \prime}(z)+\phi^{\prime}(z) \varphi^{\prime}(z)\right] f^{\prime}(\varphi(z))\right| \\
\quad+\sup _{z \in \Delta \backslash K} \mu(|z|)\left|\phi(z)\left(\varphi^{\prime}(z)\right)^{2} f^{\prime \prime}(\varphi(z))\right|
\end{aligned}
$$




$$
\begin{aligned}
& +\sup _{z \in \Delta \backslash K} \mu(|z|)\left|\left[\phi(z) \varphi^{\prime \prime}(z)+\phi^{\prime}(z) \varphi^{\prime}(z)\right] f^{\prime}(\varphi(z))\right| \\
\leq & \sup _{z \in K} \mu(|z|)\left|\phi(z)\left(\varphi^{\prime}(z)\right)^{2} f^{\prime \prime}(\varphi(z))\right|+\sup _{z \in K} \mu(|z|)\left|\left[\phi(z) \varphi^{\prime \prime}(z)+\phi^{\prime}(z) \varphi^{\prime}(z)\right] f^{\prime}(\varphi(z))\right| \\
& +\sup _{z \in \Delta \backslash K} \mu(|z|) \frac{\left|\phi(z)\left(\varphi^{\prime}(z)\right)^{2}\right|}{1-|\varphi(z)|^{2}}\left\|f_{k}\right\|_{\mathcal{Z}} \\
& +C \sup _{z \in \Delta \backslash K} \mu(|z|)\left|\phi(z) \varphi^{\prime \prime}(z)+\phi^{\prime}(z) \varphi^{\prime}(z)\right| \ln \frac{e}{1-|\varphi(z)|^{2}}\left\|f_{k}\right\|_{\mathcal{Z}} \\
\leq & C_{2} \sup _{|\omega| \leq \delta}\left|f_{k}^{\prime \prime}(\omega)\right|+C_{1} \sup _{|\omega| \leq \delta}\left|f_{k}^{\prime}(\omega)\right|+(C+1) \varepsilon\left\|f_{k}\right\|_{\mathcal{Z}},
\end{aligned}
$$

i.e. we obtain

$$
\begin{aligned}
\left\|\phi D C_{\varphi} f_{k}\right\|_{B_{\mu}} \leq & C_{2} \sup _{|\omega| \leq \delta}\left|f_{k}^{\prime \prime}(\omega)\right|+C_{1} \sup _{|\omega| \leq \delta}\left|f_{k}^{\prime}(\omega)\right| \\
& +(C+1) \varepsilon\left\|f_{k}\right\|_{\mathcal{Z}}+|\phi(0)|\left|f_{k}^{\prime}(\varphi(0))\right|\left|\varphi^{\prime}(0)\right| .
\end{aligned}
$$

Since $f_{k}$ converges to 0 uniformly on compact subsets of $\Delta$ as $k \rightarrow \infty$, from Cauchy's estimate, it follows that $f_{k}^{\prime} \rightarrow 0$ and $f_{k}^{\prime \prime} \rightarrow 0$ as $k \rightarrow \infty$ on compact subsets of $\Delta$. Hence, letting $k \rightarrow \infty$ in (2.24), and using the fact that $\varepsilon$ is an arbitrary positive number, we obtain

$$
\lim _{k \rightarrow \infty}\left\|\phi D C_{\varphi} f_{k}\right\|_{B_{\mu}}=0
$$

By combining this with Lemma 2.1 the result easily follows. The proof of Theorem 2.4 is completed.

Theorem 2.5 Let $\varphi$ be an analytic self-map of $\Delta$, and let $\phi$ be an analytic function in $\Delta$. Suppose that $\mu$ is normal. Then $\phi D C_{\varphi}: \mathcal{Z}_{0} \rightarrow B_{\mu, 0}$ is bounded if and only if $\phi D C_{\varphi}: \mathcal{Z}_{0} \rightarrow$ $B_{\mu}$ is bounded and

$$
\lim _{|z| \rightarrow 1} \mu(|z|)\left|\phi(z)\left(\varphi^{\prime}(z)\right)^{2}\right|=0 \text { and } \quad \lim _{|z| \rightarrow 1} \mu(|z|)\left|\phi(z) \varphi^{\prime \prime}(z)+\phi^{\prime}(z) \varphi^{\prime}(z)\right|=0 .
$$

Proof of Theorem 2.5 Assume that $\phi D C_{\varphi}: \mathcal{Z}_{0} \rightarrow B_{\mu, 0}$ is bounded. Then, it is clear that $\phi D C_{\varphi}: \mathcal{Z}_{0} \rightarrow B_{\mu}$ is bounded. Taking the test functions $f(z)=z$ and $f(z)=z^{2}$ respectively, we obtain (2.25).

Conversely, assume that $\phi D C_{\varphi}: \mathcal{Z}_{0} \rightarrow B_{\mu}$ is bounded and (2.25) holds. Then for each polynomial $p$, we have

$$
\begin{aligned}
& \mu(|z|)\left|\left(\phi D C_{\varphi} p\right)^{\prime}(z)\right| \\
& \quad \leq \mu(|z|)\left|\phi(z)\left(\varphi^{\prime}(z)\right)^{2} p^{\prime \prime}(\varphi(z))\right|+\mu(|z|)\left|\left[\phi(z) \varphi^{\prime \prime}(z)+\phi^{\prime}(z) \varphi^{\prime}(z)\right] p^{\prime}(\varphi(z))\right| .
\end{aligned}
$$

In view of the facts

$$
\sup _{\omega \in \Delta}\left|p^{\prime \prime}(\omega)\right|<\infty, \quad \sup _{\omega \in \Delta}\left|p^{\prime}(\omega)\right|<\infty
$$

from (2.25) and (2.26), it follows that $\phi D C_{\varphi} p \in B_{\mu, 0}$. Since the set of all polynomials is dense in $\mathcal{Z}_{0}$ (see [23]), it follows that for every $f \in \mathcal{Z}_{0}$, there is a sequence of polynomials 
$\left(p_{n}\right)_{n \in N}$ such that $\left\|f-p_{n}\right\|_{\mathcal{Z}} \rightarrow 0$ as $n \rightarrow \infty$. Hence

$$
\left\|\phi D C_{\varphi} f-\phi D C_{\varphi} p_{n}\right\|_{B_{\mu}} \leq\left\|\phi D C_{\varphi}\right\|_{\mathcal{Z}_{0} \rightarrow B_{\mu}}\left\|f-p_{n}\right\|_{\mathcal{Z}} \rightarrow 0
$$

as $n \rightarrow \infty$. Since the operator $\phi D C_{\varphi}: \mathcal{Z}_{0} \rightarrow B_{\mu}$ is bounded, we have $\phi D C_{\varphi}\left(\mathcal{Z}_{0}\right) \subseteq B_{\mu, 0}$, which implies the boundedness of $\phi D C_{\varphi}: \mathcal{Z}_{0} \rightarrow B_{\mu, 0}$.

Theorem 2.6 Let $\varphi$ be an analytic self-map of $\Delta$, and let $\phi$ be an analytic function in $\Delta$. Suppose that $\mu$ is normal. Then the following statements are equivalent.

(i) $\phi D C_{\varphi}: \mathcal{Z} \rightarrow B_{\mu, 0}$ is compact;

(ii) $\phi D C_{\varphi}: \mathcal{Z}_{0} \rightarrow B_{\mu, 0}$ is compact;

(iii)

$$
\lim _{|z| \rightarrow 1} \mu(|z|) \frac{\left|\phi(z)\left(\varphi^{\prime}(z)\right)^{2}\right|}{1-|\varphi(z)|^{2}}=0
$$

and

$$
\lim _{|z| \rightarrow 1} \mu(|z|)\left|\phi(z) \varphi^{\prime \prime}(z)+\phi^{\prime}(z) \varphi^{\prime}(z)\right| \ln \frac{e}{1-|\varphi(z)|^{2}}=0
$$

Proof of Theorem 2.6 (i) $\Rightarrow$ (ii). This implication is trivial.

(ii) $\Rightarrow$ (iii). Assume that $\phi D C_{\varphi}: \mathcal{Z}_{0} \rightarrow B_{\mu, 0}$ is compact. Then $\phi D C_{\varphi}: \mathcal{Z}_{0} \rightarrow B_{\mu, 0}$ is bounded. From the proof of Theorem 2.5, we have

$$
\lim _{|z| \rightarrow 1} \mu(|z|)\left|\phi(z) \varphi^{\prime \prime}(z)+\phi^{\prime}(z) \varphi^{\prime}(z)\right|=0
$$

and

$$
\lim _{|z| \rightarrow 1} \mu(|z|)\left|\phi(z)\left(\varphi^{\prime}(z)\right)^{2}\right|=0
$$

Hence, if $\|\varphi\|_{\infty}<1$, from (2.29) and (2.30), we obtain

$$
\lim _{|z| \rightarrow 1} \mu(|z|) \frac{\left|\phi(z)\left(\varphi^{\prime}(z)\right)^{2}\right|}{1-|\varphi(z)|^{2}} \leq \frac{1}{1-\|\varphi\|_{\infty}^{2}} \lim _{|z| \rightarrow 1} \mu(|z|)\left|\phi(z)\left(\varphi^{\prime}(z)\right)^{2}\right|=0
$$

and

$$
\begin{aligned}
& \lim _{|z| \rightarrow 1} \mu(|z|)\left|\phi(z) \varphi^{\prime \prime}(z)+\phi^{\prime}(z) \varphi^{\prime}(z)\right| \ln \frac{e}{1-|\varphi(z)|^{2}} \\
& \quad \leq \ln \frac{e}{1-\|\varphi\|_{\infty}^{2}} \lim _{|z| \rightarrow 1} \mu(|z|)\left|\phi(z) \varphi^{\prime \prime}(z)+\phi^{\prime}(z) \varphi^{\prime}(z)\right|=0,
\end{aligned}
$$

from which the result follows in this case.

Now assume that $\|\varphi\|_{\infty}=1$. Let $\left(z_{k}\right)_{k \in N}$ be a sequence such that $\left|\varphi\left(z_{k}\right)\right| \rightarrow 1$ as $k \rightarrow \infty$. Since $\phi D C_{\varphi}: \mathcal{Z}_{0} \rightarrow B_{\mu}$ is compact, by Theorem 2.4, we have

$$
\lim _{|\varphi(z)| \rightarrow 1} \mu(|z|) \frac{\left|\phi(z)\left(\varphi^{\prime}(z)\right)^{2}\right|}{1-|\varphi(z)|^{2}}=0
$$


and

$$
\lim _{|\varphi(z)| \rightarrow 1} \mu(|z|)\left|\phi(z) \varphi^{\prime \prime}(z)+\phi^{\prime}(z) \varphi^{\prime}(z)\right| \ln \frac{e}{1-|\varphi(z)|^{2}}=0
$$

From (2.30) and (2.31), it follows that for every $\varepsilon>0$, there exists an $r \in(0,1)$ such that $\mu(|z|) \frac{\left|\phi(z)\left(\varphi^{\prime}(z)\right)^{2}\right|}{1-|\varphi(z)|^{2}}<\varepsilon$, when $r<|\varphi(z)|<1$, and there exists a $\sigma \in(0,1)$ such that $\mu(|z|)\left|\phi(z)\left(\varphi^{\prime}(z)\right)^{2}\right| \leq \varepsilon\left(1-r^{2}\right)$, when $\sigma<|z|<1$. Therefore, when $\sigma<|z|<1$ and $r<|\varphi(z)|<$ 1 , we have

$$
\mu(|z|) \frac{\left|\phi(z)\left(\varphi^{\prime}(z)\right)^{2}\right|}{1-|\varphi(z)|^{2}}<\varepsilon
$$

On the other hand, if $\sigma<|z|<1$ and $|\varphi(z)| \leq r$, we obtain

$$
\mu(|z|) \frac{\left|\phi(z)\left(\varphi^{\prime}(z)\right)^{2}\right|}{1-|\varphi(z)|^{2}}<\mu(|z|) \frac{\left|\phi(z)\left(\varphi^{\prime}(z)\right)^{2}\right|}{1-r^{2}}<\varepsilon .
$$

Inequality (2.33) together with (2.34) gives the (2.27). Similarly, (2.29) and (2.32) imply (2.28).

(iii) $\Rightarrow$ (i). Let $f \in \mathcal{Z}$. Then we have

$$
\begin{aligned}
& \mu(|z|)\left|\left(\phi D C_{\varphi} f\right)^{\prime}(z)\right| \\
& \quad \leq C\left[\mu(|z|) \frac{\left|\phi(z)\left(\varphi^{\prime}(z)\right)^{2}\right|}{1-|\varphi(z)|^{2}}+\mu(|z|)\left|\phi(z) \varphi^{\prime \prime}(z)+\phi^{\prime}(z) \varphi^{\prime}(z)\right| \ln \frac{e}{1-|\varphi(z)|^{2}}\right]\|f\|_{\mathcal{Z}} .
\end{aligned}
$$

Taking the supremum in this inequality over all $f \in \mathcal{Z}$ such that $\|f\|_{\mathcal{Z}} \leq 1$, then letting $|z| \rightarrow 1$, and using (2.27) and (2.28), we obtain

$$
\lim _{|z| \rightarrow 1} \sup _{\|f\|_{\mathcal{Z}} \leq 1} \mu(|z|)\left|\left(\phi D C_{\varphi} f\right)^{\prime}(z)\right|=0
$$

From Lemma 2.2 it follows that the operator $\phi D C_{\varphi}: \mathcal{Z} \rightarrow B_{\mu, 0}$ is compact.

Similarly to the proofs of Theorems 2.3-2.6, we can get the following results; we omit the proof.

Theorem 2.7 Let $\varphi$ be an analytic self-map of $\Delta$, and let $\phi$ be an analytic function in $\Delta$. Suppose that $\mu$ is normal. Then the following statements are equivalent.

(i) $\phi C_{\varphi} D: \mathcal{Z} \rightarrow B_{\mu}$ is bounded;

(ii) $\phi C_{\varphi} D: \mathcal{Z}_{0} \rightarrow B_{\mu}$ is bounded;

(iii)

$$
\sup _{z \in \Delta} \mu(|z|) \frac{\left|\phi(z) \varphi^{\prime}(z)\right|}{1-|\varphi(z)|^{2}}<\infty
$$

and

$$
\sup _{z \in \Delta} \mu(|z|)\left|\phi^{\prime}(z)\right| \ln \frac{e}{1-|\varphi(z)|^{2}}<\infty
$$


Theorem 2.8 Let $\varphi$ be an analytic self-map of $\Delta$, and let $\phi$ be an analytic function in $\Delta$. Suppose that $\mu$ is normal. Then the following statements are equivalent.

(i) $\phi C_{\varphi} D: \mathcal{Z} \rightarrow B_{\mu}$ is compact;

(ii) $\phi C_{\varphi} D: \mathcal{Z}_{0} \rightarrow B_{\mu}$ is compact;

(iii) $\phi C_{\varphi} D: \mathcal{Z} \rightarrow B_{\mu}$ is bounded,

$$
\lim _{|\varphi(z)| \rightarrow 1} \mu(|z|) \frac{\left|\phi(z) \varphi^{\prime}(z)\right|}{1-|\varphi(z)|^{2}}=0
$$

and

$$
\lim _{|\varphi(z)| \rightarrow 1} \mu(|z|)\left|\phi^{\prime}(z)\right| \ln \frac{e}{1-|\varphi(z)|^{2}}=0
$$

Theorem 2.9 Let $\varphi$ be an analytic self-map of $\Delta$, and let $\phi$ be an analytic function in $\Delta$. Suppose that $\mu$ is normal. Then $\phi C_{\varphi} D: \mathcal{Z}_{0} \rightarrow B_{\mu, 0}$ is bounded if and only if $\phi C_{\varphi} D: \mathcal{Z}_{0} \rightarrow$ $B_{\mu}$ is bounded and

$$
\lim _{|z| \rightarrow 1} \mu(|z|)\left|\phi(z) \varphi^{\prime}(z)\right|=0 \quad \text { and } \quad \lim _{|z| \rightarrow 1} \mu(|z|)\left|\phi^{\prime}(z)\right|=0
$$

Theorem 2.10 Let $\varphi$ be an analytic self-map of $\Delta$, and let $\phi$ be an analytic function in $\Delta$. Suppose that $\mu$ is normal. Then the following statements are equivalent.

(i) $\phi C_{\varphi} D: \mathcal{Z} \rightarrow B_{\mu, 0}$ is compact;

(ii) $\phi C_{\varphi} D: \mathcal{Z}_{0} \rightarrow B_{\mu, 0}$ is compact;

(iii)

$$
\lim _{|z| \rightarrow 1} \mu(|z|) \frac{\left|\phi(z) \varphi^{\prime}(z)\right|}{1-|\varphi(z)|^{2}}=0
$$

and

$$
\lim _{|z| \rightarrow 1} \mu(|z|)\left|\phi^{\prime}(z)\right| \ln \frac{e}{1-|\varphi(z)|^{2}}=0
$$

\section{Competing interests}

The authors declare that they have no competing interests.

Authors' contributions

All authors contributed equally to the manuscript. All authors read and approved the final manuscript.

\section{Acknowledgements}

The authors would like to thank the referees for their valuable suggestions which greatly improve the present article. The work was supported by the United Technology Foundation of Science and Technology Department of Guizhou Province and Guizhou Normal University (Grant No. LKS[2012]12), and National Natural Science Foundation of China (Grant No.

11171080, Grant No. 11171277)

\section{Received: 12 January 2014 Accepted: 8 April 2014 Published: 02 May 2014}

\section{References}

1. Zhu, K: Bloch type spaces of analytic functions. Rocky Mt. J. Math. 23(3), 1143-1177 (1993)

2. Shields, A, Williams, D: Bonded projections, duality, and multipliers in spaces of analytic functions. Trans. Am. Math. Soc. 162, 287-302 (1971)

3. Fu, X, Zhu, X: Weighted composition operators on some weighted spaces in the unit ball. Abstr. Appl. Anal. 2008, Article ID 605807 (2008) 
4. Hu, Z, Wang, S: Composition operators on Bloch-type spaces. Proc. R. Soc. Edinb. A 135(6), 1229-1239 (2005)

5. Krantz, SG, Stević, S: On the iterated logarithmic Bloch space on the unit ball. Nonlinear Anal. TMA 71(5-6), 1772-1795 (2009)

6. Duren, P: Theory of $H^{P}$ Spaces. Academic Press, New York (1970)

7. Li, S, Stević, S: Volterra type operators on Zygmund spaces. J. Inequal. Appl. 2007, Article ID 32124 (2007)

8. Stević, S: On an integral operator from the Zygmund space to the Bloch-type space on the unit ball. Glasg. Math. J. 51(2), 275-287 (2009)

9. Stević, S: On an integral-type operator from Zygmund-type spaces to mixed-norm spaces on the unit ball. Abstr. Appl. Anal. 2010, Article ID 198608 (2010)

10. Cowen, C, Maccluer, B: Composition Operators on Spaces of Analytic Functions. Studies in Advanced Mathematics. CRC Press, Boca Raton (1995)

11. Li, S, Stević, S: Generalized composition operators on Zygmund spaces and Bloch type spaces. J. Math. Anal. Appl. 338(2), 1282-1295 (2008)

12. Madigan, K, Matheson, A: Compact composition operators on the Bloch space. Trans. Am. Math. Soc. 347, 2679-2687 (1995)

13. Shapiro, J: Composition Operators and Classical Function Theory. Springer, New York (1993)

14. Stević, S: Generalized composition operators between mixed norm space and some weighted spaces. Numer. Funct. Anal. Optim. 29, 426-434 (2009)

15. Stević, S: Norm of weighted composition operators from Bloch space to $H_{\mu}^{\infty}$ on the unit ball. Ars Comb. 88, 125-127 (2008)

16. Stević, S: Norm of weighted composition operators from $\alpha$-Bloch spaces to weighted-type spaces. Appl. Math. Comput. 215, 818-820 (2009)

17. Ueki, S: Composition operators on the Privalov spaces of the unit ball of $\mathbb{C}^{n}$. J. Korean Math. Soc. 42(1), 111-127 (2005)

18. Ueki, S: Weighted composition operators on the Bargmann-Fock space. Int. J. Mod. Math. 3(3), 231-243 (2008)

19. Zhu, X: Generalized weighted composition operators from Bloch-type spaces to weighted Bergman spaces. Indian J. Math. 49(2), 139-149 (2007)

20. Hibschweiler, R, Portnoy, N: Composition followed by differentiation between Bergman and Hardy spaces. Rocky Mt. J. Math. 35(3), 843-855 (2005)

21. Li, S, Stević, S: Composition followed by differentiation between Bloch type spaces. J. Comput. Anal. Appl. 9(2), 195-205 (2007)

22. Li, S, Stević, S: Composition followed by differentiation between $H^{\infty}$ and $\alpha$-Bloch spaces. Houst. J. Math. 35(1), 327-340 (2009)

23. Li, S, Stević, S: Products of composition and differentiation operators from Zygmund spaces to Bloch spaces and Bers spaces. Appl. Math. Comput. 217, 3144-3154 (2010)

24. Li, S, Stević, S: Composition followed by differentiation from mixed-norm spaces to $\alpha$-Bloch spaces. Sb. Math. 199(12), 1847-1857 (2008)

25. Long, J, Wu, P: Weighted composition followed and proceeded by differentiation operators from $Q_{k}(p, q)$ spaces to Bloch-type spaces. J. Inequal. Appl. 2012, Article ID 160 (2012)

26. Ohno, S: Products of composition and differentiation between Hardy spaces. Bull. Aust. Math. Soc. 73(2), 235-243 (2006)

27. Stević, S: Weighted differentiation composition operators from mixed-norm spaces to weighted-type spaces. Appl. Math. Comput. 211, 222-233 (2009)

28. Stević, S: Norm and essential norm of composition followed by differentiation from $\alpha$-Bloch spaces to $H_{\mu}^{\infty}$. Appl. Math. Comput. 207(1), 225-229 (2009)

29. Stević, S: Products of composition and differentiation operators on the weighted Bergman space. Bull. Belg. Math. Soc. Simon Stevin 16(4), 623-635 (2009)

30. Fu, X, Li, S: Composition operators from Zygmund spaces into QK spaces. J. Inequal. Appl. 2013, Article ID 175 (2013)

31. Li, S, Stević, S: On an integral-type operator from $\omega$-Bloch spaces to $\mu$-Zygmund spaces. Appl. Math. Comput. 215(12), 4385-4391 (2010)

32. Li, S, Stević, S: Weighted composition operators from Zygmund spaces into Bloch spaces. Appl. Math. Comput. 206(2), 825-831 (2008)

33. Li, S, Stević, S: Integral-type operators from Bloch-type spaces to Zygmund-type spaces. Appl. Math. Comput. 215 464-473 (2009)

34. Li, S, Stević, S: Products of Volterra type operator and composition operator from $H^{\infty}$ and Bloch spaces to the Zygmund space. J. Math. Anal. Appl. 345, 40-52 (2008)

35. Liu, Y, Yu, Y: Riemann-Stieltjes operator from mixed norm spaces to Zygmund-type spaces on the unit ball. Taiwan. J. Math. 17(5), 1751-1764 (2013)

36. Yang, C: Integral-type operators from $F(p ; q ; s)$ spaces to Zygmund-type spaces on the unit ball. J. Inequal. Appl. 2010 Article ID 789285 (2010)

37. Zhu, X: Extended Cesàro operators from mixed norm spaces to Zygmund type spaces. Tamsui Oxford Univ. J. Math. Sci. 26(4), 411-422 (2010) 